Discrete Comput Geom 37:287-296 (2007)

DOI: $10.1007 / \mathrm{s} 00454-006-1293-4$

\title{
Sommerville's Missing Tetrahedra
}

\author{
Allan L. Edmonds \\ Department of Mathematics, Indiana University, \\ Bloomington, IN 47405, USA \\ edmonds@indiana.edu
}

\begin{abstract}
D. M. Y. Sommerville's 1923 classification of tetrahedra that can tile 3-space in a proper, face-to-face manner is completed, by showing that the case he failed to consider cannot occur, although it does occur in non-proper and in non-face-to-face tilings.
\end{abstract}

\section{Introduction}

Which tetrahedra tile euclidean 3-space? This question has a long and somewhat complicated history, going back to Aristotle, who incorrectly thought that the regular tetrahedron could tile space.

More recent work goes back to Sommerville [10], who made the first systematic study of tetrahedra that can tile euclidean 3-space in the early twentieth century. (His work was presaged by work of Hill [7] in 1896 on determining which tetrahedra are congruent by dissection to cubes and by work in crystallography. It is known that a tetrahedron that tiles euclidean 3 -space is congruent by dissection to a cube.)

Sommerville discovered a list of exactly four tilings (up to isometry and re-scaling) and apparently thought that he had given a complete list, under the assumptions, which we follow here unless stated otherwise, that all tiles are properly congruent (i.e., congruent by an orientation-preserving isometry) and meet face-to-face. However, there turned out to be a curious gap at the end of his argument, where he failed to analyze the final case out of eleven he enumerated. See Section 9 for more discussion of the Sommerville paper.

The subject received further attention much later when more families of tilers in dimension 3 were discovered or re-discovered, including ones that may not meet faceto-face. Compare [1] and [6]. In the non-proper and non-face-to-face situations there are infinite families of non-similar tetrahedral tilers. By contrast this paper completes the proof that in the proper, face-to-face context there are only four tetrahedral tilers up to similarity. 
We refer to the article of Senechal [9] for the history of these questions up to about 1980 and to Debrunner [3] for slightly more recent history coupled with a higher-dimensional perspective.

The particular tilings constructed by Sommerville all appear to admit a group of symmetries acting transitively on the tiles. See the discussion in [4], where tile-transitive tilings by tetrahedra are completely enumerated. There it is concluded that all such tiletransitive tilings arise in an appropriate sense from the basic Sommerville construction of triangulating a triangular prism. Also, see [4] for recent work involving more general tilings by other "topological Platonic solids," as well as further references to the literature on tile-transitive and face-transitive tilings. In this paper, however, we make no assumptions about the existence of any symmetry.

In recent years the subject of tilings has received a certain impetus from computer graphics and other computer applications where it is helpful to produce fine-mesh decompositions of regions for numerical routines that struggle in the presence of obtuse angles. In fact, the author of the present paper was attracted to the problem addressed here by the comment of Eppstein et al. [5, end of Section 3] that "... it seems likely that there is no tiling of space by congruent acute tetrahedra...." These authors skirted the issue by constructing tilings that involve several shapes of acute tetrahedra, rather than just one.

In an attempt to address this question while bypassing questions of the completeness of Sommerville's analysis, Kiernan [8] proved the following result, which would be a corollary of Sommerville's claim: No tetrahedron in which all six dihedral angles are acute admits a proper, face-to-face tiling of euclidean 3-space. Her proof uses a delicate case-by-case analysis, not unlike that of Sommerville.

In this paper we deal directly with Sommerville's missing case (xi).

By a Sommerville case (xi) tetrahedron we mean a tetrahedron that contains three edges of length $p$ in a chain and three edges of length $q$ in a chain. We say that such a Sommerville case (xi) tetrahedron has type $p / q$. It is characterized up to isometry by the unordered pair $\{p, q\}$ and up to similarity by the ratio $p / q$ (or its multiplicative inverse). By simple re-scaling we sometimes assume that $q=1$. The Sommerville case (xi) tetrahedra are also characterized as those tetrahedra all of whose facets are isosceles but which do not admit a mirror symmetry. To fix notation that we preserve throughout this paper we assume that $T=A B C D$ is a tetrahedron in euclidean 3-space such that $|A B|=|B C|=|C D|=p$ and $|B D|=|D A|=|A C|=q$. The main result of this paper is the following.

Theorem 1.1. No Sommerville case (xi) tetrahedron is a tile of a proper, face-to-face tiling of euclidean 3-space.

The key technique is the derivation and use of explicit formulas for the dihedral angles in such a tetrahedron.

Corollary 1.2. Sommerville's list [10] of proper, face-to-face tilers in dimension 3 is complete.

We further show that exactly one of Sommerville's case (xi) tetrahedra can tile, when one allows tilings that are neither face-to-face nor proper. It turns out to be a particular 
member of Goldberg's first family constructed in [6]. Goldberg's description makes it clear that it does indeed tile improperly face-to-face and also tiles properly non-face-toface.

\section{Tiling Definitions}

A tetrahedral tiling consists of a collection $\mathcal{T}=\left\{T_{i}\right\}$ of tetrahedra in $\mathbb{R}^{3}$ such that

(1) $\bigcup T_{i}=\mathbb{R}^{3}$ and

(2) any two tetrahedra in $\mathcal{T}$ have disjoint interiors.

The tiling is face-to-face if the intersection of any two of the tetrahedra is either empty or consists exactly of a vertex, an edge, or a face of both of them. In this paper we are only concerned with face-to-face tilings.

Given a tetrahedron $T$ in $\mathbb{R}^{3}$, we say that $T$ tiles if there is a face-to-face tetrahedral tiling in which each tetrahedron is congruent to $T$.

We say that $T$ properly tiles if there is a face-to-face tetrahedral tiling in which each tetrahedron is properly congruent to $T$. That is, for each $T_{i} \in \mathcal{T}$ there is an orientationpreserving isometry of $\mathbb{R}^{3}$ taking $T_{i}$ to $T$. We say that $T$ is a proper tiler.

\section{The Known Tetrahedral Tilers}

We refer to the papers of Dubrunner [3] and Goldberg [6] for tabulation of the known tilers.

An inspection of Goldberg's tables shows that there is exactly one tetrahedron on his list, that by special choice of a parameter corresponds to a Sommerville case (xi) tetrahedron. In Goldberg's first family, using his notation, we may insist that $c=3 a$, in which case the tetrahedron is of the correct type. The others have too many different edge lengths or edge lengths in the wrong pattern. If we set $c=3 a$, a little calculation yields the type $p / q$, where $q=c=\sqrt{9 / 5} e, p=b=\sqrt{6 / 5} e$, so that $p / q=\sqrt{2 / 3}$. In Goldberg's nomenclature, this is the tetrahedron $H(1 / 3)$.

It turns out to be exactly the possibility that we locate in Section 7 when we ask which Sommerville case (xi) tetrahedra tile non-properly. Goldberg's construction makes it clear that this tetrahedron does indeed tile non-properly and face-to-face or properly and not face-to-face.

Goldberg also records the dihedral angles explicitly in terms of the edge lengths, which provides a useful check on the general formulas derived in Section 5.

\section{The Dihedral Angles of a General Tetrahedron}

Let $A B C D$ be a tetrahedon in 3-space. Associated with each of the six edges is a dihedral angle, the ordinary angle between the two planar faces incident at the edge. It is measured strictly between 0 and $\pi$ radians. We denote the dihedral angle along edge $A B$ by $\angle A B$. 
Where no confusion arises we do not distinguish notationally between an angle and its measure.

Sum of the Dihedral Angles at a Vertex. The three dihedral angles at any vertex have sum greater than $\pi$. One way to understand this is to note that the intersection of a tetrahedron with a small sphere (scaled to have radius 1 for convenience) centered at a vertex yields a spherical triangle whose angles are the dihedral angles incident at that vertex. The angles of a spherical triangle add up to more than $\pi$, since the defect, the sum of the angles minus $\pi$, measures the area of the triangle.

Sum of the Dihedral Angles of a Tetrahedron. The six dihedral angles of a tetrahedron have sum greater than $2 \pi$. One can see this by adding up the dihedral angles at all four vertices to obtain a sum greater than $4 \pi$, but this sum counts each dihedral angle twice.

Basic Edge Equations. If $T$ tiles face-to-face, then for any edge of length $r$, say, in the tiling, the dihedral angles around that edge sum to $2 \pi$. This sum is a non-negative integer combination of the dihedral angles of $T$ along its edges of length $r$.

\section{The Dihedral Angles of Sommerville Case (xi) Tetrahedra}

The goal of this section is to derive explicit formulas for the dihedral angles of a Sommerville case (xi) tetrahedron of type $p / q$. Such a tetrahedron has two kinds of faces: two isosceles triangles with two edges of length $p$ and one edge of length $q$, and two isosceles triangles with two edges of length $q$ and one edge of length $p$. Applying the triangle inequality to the faces we note the necessary condition that

$$
\frac{1}{2}<p / q<2 .
$$

In order for the tetrahedron of type $p / q$ to exist, however, the stronger necessary and sufficient condition

$$
0.618 \approx \frac{\sqrt{5}-1}{2}<\frac{p}{q}<\frac{\sqrt{5}+1}{2} \approx 1.618
$$

holds. (The limiting bounds correspond to configurations where all four facets lie flat in the plane, forming a trapezoid of sides $q, q, q, p$ and two diagonals of length $p$ or a trapezoid of sides $p, p, p, q$ and two diagonals of length $q$.) One can derive this from first principles by calculating the maximum and minimum distance between opposite vertices of two isosceles triangles with edges of lengths $p, p, q$ and $q, q, p$, respectively, and one edge in common. This also follows from a standard condition for realizing given edge lengths by a simplex, as in, for example, [2]. 
Note that if $A B C D$ is a Sommerville case (xi) tetrahedron of type $p / q$ with $|A B|=$ $|B C|=|C D|=p$ and $|C A|=|A D|=|D B|=q$, consideration of the proper congruence interchanging vertices $A$ and $D$ and vertices $B$ and $C$, show that the dihedral angles along the edges $A B$ and $C D$ are equal, as are the dihedral angles along $C A$ and $D B$.

Proposition 5.1. Let $A B C D$ be a Sommerville tetrahedron of type $p / q$ with $|A B|=$ $|B C|=|C D|=p$ and $|C A|=|A D|=|D B|=q$. Then the dihedral angles along the edges are given by

$$
\begin{gathered}
\left.\cos \angle C A=\cos \angle D B=\frac{q\left(3 p^{2}-2 q^{2}\right)}{p \sqrt{\left(4 p^{2}-q^{2}\right)\left(4 q^{2}-p^{2}\right)}} \quad \text { (end edges of length } q\right), \\
\left.\cos \angle A B=\cos \angle C D=\frac{p\left(3 q^{2}-2 p^{2}\right)}{q \sqrt{\left(4 p^{2}-q^{2}\right)\left(4 q^{2}-p^{2}\right)}} \quad \text { (end edges of length } p\right), \\
\cos \angle B C=\frac{\left(p^{2}-q^{2}\right)^{2}+p^{4}}{q^{2}\left(4 p^{2}-q^{2}\right)} \quad \text { (middle edge of length } p \text { ) }
\end{gathered}
$$

and

$$
\cos \angle A D=\frac{\left(p^{2}-q^{2}\right)^{2}+q^{4}}{p^{2}\left(4 q^{2}-p^{2}\right)} \quad \text { (middle edge of length } q \text { ). }
$$

Proof. This is an elementary, but perhaps tedious, exercise in three-dimensional coordinate geometry. We omit the details and instead discuss only the strategy of the computation.

It suffices to compute only the first and third in the list of four. The second and fourth can be obtained by reversing the role of $p$ and $q$ in the first and third.

Our approach is to view the tetrahedron as given by two adjacent facets, with a variable dihedral angle between them, allowed to vary from 0 to $\pi$. We then compute the distance between the two vertices that determine the remaining edge, as a function of the given edge lengths of the two triangles and the cosine of the dihedral angle they make. Finally we set this edge length equal to $p$ and solve for the dihedral angle.

Things simplify at least a little to assume the two given triangles are isosceles with edge lengths $(p, p, q)$ and $(p, q, q)$. It is convenient to choose coordinates so that the two triangles start out in the $x y$-plane, corresponding to angle $=\pi$, with the common edge on the $y$-axis and its midpoint at the origin.

Further details of the distance calculation are left to the reader. Fortunately at the end it is easy to solve for the desired cosine.

As a quick check note that if one sets $q=p$, then this gives the standard result $\cos \theta=\frac{1}{3}$ for the dihedral angle of an equilateral tetrahedron. It also agrees with the calculation of dihedral angles given by Goldberg [6] in the case of the one Sommerville case (xi) tetrahedron in his tables.

Using the formulas above one can make several useful observations. Angles $A C$ and $B D$ are acute if and only if $p / q>\sqrt{2 / 3}$. Dually, angles $A B$ and $D C$ are acute if and only if $q / p>\sqrt{2 / 3}$. On the other hand, angles $A D$ and $B C$ are always acute. 


\section{Proper Face-to-Face Tilings}

Here we apply the calculation of the dihedral angles in a Sommerville case (xi) tetrahedron.

Theorem 6.1. A Sommerville case (xi) tetrahedron does not tile 3-space in a proper, face-to-face manner.

Proof. The key observation is that for any proper, face-to-face tiling by a Sommerville case (xi) tetrahedron, the tiling is completely determined by a single tile. If $T$ is such a tile, and $F$ is any facet of $T$, then the tile adjacent to $T$ across $F$ is obtained uniquely by rotating $T$ by $180^{\circ}$ in space around the axis given by the line of symmetry of $F$. One cannot reflect $T$ across $F$ since $T$ is not properly congruent to its mirror image. Moreover, there is a proper congruence of $T$ itself interchanging pairs of facets of the same type.

From this observation it follows that in any edge equation corresponding to one of the two edge lengths, the dihedral angle corresponding to an "end edge" appears twice for each appearance of the dihedral angle corresponding to the "middle edge." One way to visualize this is to note that, up to rotations fixing the $z$-axis, there are exactly three ways to position $T$ in 3 -space so that an edge of length $p$, say, lies on the positive $z$-axis starting at the origin. Moreover, the required chain of tetrahedra cycles through these three positions one after the other.

In our usual notation, let $A B C D$ be a Sommerville case (xi) tetrahedron, with $|A B|=$ $|B C|=|C D|=p$ and $|C A|=|A D|=|D B|=q$, such that $A B C D$ tiles 3-space. Then we obtain

$$
k(\angle A B+\angle B C+\angle C D)=2 \pi
$$

and

$$
\ell(\angle C A+\angle A D+\angle D B)=2 \pi
$$

for some positive integers $k$ and $\ell$. (Again, there is no real distinction between $A B$ and $C D$ or between $C A$ and $D B$, since these pairs of edges are related by a proper congruence.)

If both $k, \ell \geq 2$, then we find that

$$
\angle A B+\angle B C+\angle C D+\angle C A+\angle A D+\angle D B \leq 2 \pi .
$$

However, this contradicts the known fact that the six dihedral angles in a tetrahedron add up to more than $2 \pi$.

We conclude that $k$ or $\ell=1$. Without loss of generality assume that $k=1$. Then

$$
\angle A B+\angle B C+\angle C D=2 \pi
$$

Now we have explicit formulas for these angles in terms of $p$ and $q$. We may normalize so that $q=1$. The calculation in the preceding section provides an explicit expression of this function as

$$
f(p)=2 \arccos \left(\frac{p\left(3-2 p^{2}\right)}{\sqrt{\left(4 p^{2}-1\right)\left(4-p^{2}\right)}}\right)+\arccos \left(\frac{\left(p^{2}-1\right)^{2}+p^{4}}{4 p^{2}-1}\right) .
$$


The question, then, is whether there is a value of $p$ that is realizable by a Sommerville case (xi) tetrahedron of type $p / 1$ and such that $f(p)=2 \pi$.

Graphing this rather complicated but well-behaved function in Maple or Mathematica over the required interval $(\sqrt{5}-1) / 2<p<(\sqrt{5}+1) / 2$ shows a strictly increasing function bounded between 0 and $2 \pi$. In particular, it never achieves the value $2 \pi$. (The two limiting degenerate cases correspond to when the entire tetrahedron lies flat in a plane, forming a trapezoid with sides $q, q, q, p$ or $p, p, p, q$, as indicated earlier in Section 5.) This contradicts the assumption that $A B C D$ is a proper, face-to-face tiler, and hence completes the proof.

Remark 1. Given the function $f$, which is somewhat complicated, but quite wellbehaved (from the point of view of elementary calculus), it seems acceptable to rely on the robust graphical capabilities of a computer algebra program such as Maple or Mathematica. On the other hand, one could eliminate reliance on the graph entirely by showing directly that $f^{\prime}(p)>0$ and that $f(p)<2 \pi$ for $p$ in the range that corresponds to realizable tetrahedra. Note, however, that it is not the case that both constituent summands of $f$ are separately increasing.

As indicated earlier, this completes Sommerville's analysis by ruling out the case that he failed to consider.

Corollary 6.2. Sommerville's list of four tetrahedra that tile 3-space in a proper, faceto-face manner is complete, up to similarity.

\section{Non-Proper Face-to-Face Tilings}

If one considers non-proper tilings, then there are more possibilities for angles that sum to $2 \pi$. It turns out that there is an essentially unique Sommerville case (xi) tetrahedron that tiles non-properly, but face-to-face.

Theorem 7.1. If a Sommerville case (xi) tetrahedron of type $p / q$ tiles euclidean 3space in a face-to-face, not necessarily proper, manner, then $p / q=\sqrt{2 / 3}$ or $\sqrt{3 / 2}$.

As pointed out before, Goldberg's construction shows that the Sommerville case (xi) tetrahedron of type $p / q=\sqrt{2 / 3}$ or $\sqrt{3 / 2}$ does indeed tile 3-space in a non-proper manner.

We have already ruled out a proper face-to-face tiling, so our focus here is on nonproper tilings. This result is admittedly of somewhat limited interest, and the proof is rather more difficult than that of Theorem 6.1, although in the same spirit. We therefore limit ourselves to a few remarks on the proof, highlighting the key ideas.

Around an edge of length $p$ (respectively $q$ ) the dihedral angles of tiles incident at the edge must add up to $2 \pi$, so we obtain equations of the form

$$
x \angle A B+y \angle B C=2 \pi \quad \text { and } \quad z \angle C A+w \angle A D=2 \pi,
$$


where $x, y, z, w$ represent non-negative integers and each of the four angles occurs in at least one such equation with non-zero coefficient. We normalize by scaling so that $q=1$, and study these equations as functions of $p$, where $x, y, z, w$ are non-negative integers.

Note that from our explicit calculation of the angles in a Sommerville case (xi) tetrahedron, it follows that if $p$ satisfies $x \angle A B+y \angle B C=2 \pi$, then $1 / p$ satisfies $x \angle C A+$ $y \angle A D=2 \pi$ and vice versa.

Lemma 7.2. If $x \geq 2$ and $x+y \geq 6$ then $x \angle A B+y \angle B C=2 \pi$ has a unique solution $p$ and it satisfies $p<1$. Dually, if $z \geq 2$ and $z+w \geq 6$, then $z \angle C A+w \angle A D=2 \pi$ has a unique solution $p$ and it satisfies $p>1$.

One can prove this by examining Maple or Mathematica graphs of the corresponding angle sums as functions of $p$.

Lemma 7.3. An edge equation of the form $x \angle A B+y \angle B C=2 \pi$ cannot have $x=1$. If all edge equations involving dihedral angle $\angle B C$ contain no $\angle A B$, then there is also an edge equation involving only angle $\angle A B$. Similarly, an edge equation of the form $z \angle C A+w \angle A D=2 \pi$ cannot have $z=1$. If all edge equations involving dihedral angle $\angle A D$ contain no $\angle C A$, then there is also an edge equation involving only angle $\angle C A$.

The conclusion from Lemma 7.3 is that we either have an equation of the form

$$
x \angle A B+y \angle B C=2 \pi, \quad x \geq 2,
$$

or we have a pair of equations

$$
x \angle A B=2 \pi \quad \text { and } \quad y \angle B C=2 \pi,
$$

where $y$ is even. (If $x$ is also even, then we could concoct an equation of the first sort out of a pair of equations of the second sort.) A similar statement holds for equations involving $\angle C A$ and $\angle A D$. Thus the existence of a tiling leads to at least one of the following four cases:

(1) $x \angle A B+y \angle B C=2 \pi$ and $z \angle C A+w \angle A D=2 \pi, x \geq 2, y \geq 1, z \geq 2, w \geq 1$.

(2) $x \angle A B+y \angle B C=2 \pi$ and $z \angle C A=2 \pi$ and $w \angle A D=2 \pi, x \geq 2, y \geq 1, z \geq$ $3, w \geq 4, w$ even.

(3) $x \angle A B=2 \pi$ and $y \angle B C=2 \pi$ and $z \angle C A+w \angle A D=2 \pi, x \geq 3, y \geq 4, y$ even, $z \geq 2, w \geq 1$.

(4) $x \angle A B=2 \pi$ and $y \angle B C=2 \pi$ and $z \angle C A=2 \pi$ and $w \angle A D=2 \pi, x \geq 3, y \geq$ 4, $y$ even, $z \geq 3, w \geq 4, w$ even.

Consider Case (1). We play the two types of equations off against each other. By plotting the quantities $x \angle A B+y \angle B C$ as functions of $p$ for successive values of the integers $x$ and $y$, one can see that the equation $x \angle A B+y \angle B C=2 \pi$ with $x \geq 2$, has no solution for $y=1$ or $y=2$. It has a unique solution $p=\sqrt{2 / 3}$ when $x=2$ and $y=3$, which is the case where angles $\angle A B$ and $\angle C D$ are right angles and corresponds 
to the Goldberg tetrahedron $H(1 / 3)$ mentioned in Section 3, and known to tile in a non-proper fashion. Otherwise $x \geq 2$ and $x+y \geq 6$. In this case, by Lemma 7.2, the function $x \angle A B+y \angle B C$ as a function of $p$ is greater than $2 \pi$ for $p<1$ and the equation $x \angle A B+y \angle B C=2 \pi$ has a unique solution with $p>1$.

Similarly the equation $z \angle C A+w \angle A D=2 \pi$, with $x \geq 2$ has no solution with $w=1$ or $w=2$. It has a unique solution $p=\sqrt{3 / 2}$ when $z=2$ and $w=3$, again giving the case where angles $\angle C A$ and $\angle D B$ are right angles corresponding to the Goldberg tetrahedron $H(1 / 3)$. Otherwise $z \geq 2$ and $z+w \geq 6$. In this case the equation $z \angle C A+w \angle A D=2 \pi$ has a unique solution with $p<1$.

We conclude in the first case that the only values of $p$ that satisfy both of our pair of equations are exactly the known values that give the Goldberg tetrahedron $H(1 / 3)$.

In the remaining three cases one similarly systematically rules out any solution whatsoever.

Further details are omitted.

\section{Non-Face-to-Face Tilings}

In fact the calculations in the previous section imply that no Sommerville case (xi) tetrahedron besides the Goldberg $H(1 / 3)$ case tiles 3 -space in any way whatsoever. In such a tetrahedron of type $p / q$ tiles, then the only thing that changes is that some of the edge equations now take the form

$$
x \angle A B+y \angle B C=\pi
$$

when an edge (of length $p$, say) of one tetrahedral tile crosses the interior of a facet of another tile. However, such an equation leads then to an equation

$$
2 x \angle A B+2 y \angle B C=2 \pi
$$

of the sort considered before, with even more restrictions on the coefficients. We conclude there are no further solutions to be found in this case.

\section{Concluding Remarks}

What did Sommerville actually do? There seems to be no way to know for sure. To the best of this author's knowledge, he published just two papers on the subject of tilings [10], [11], both done at essentially the same time, and apparently never returned to the subject. The second paper [11] is more descriptive and pedagogical in flavor, and shows how the tilers he found arise by suitably triangulating a triangular prism. The former paper [10] makes no broad claims at the beginning. He merely announces a systematic study of tilings in dimensions two and three, allowing for elliptic and hyperbolic as well as euclidean geometry, which he treats together. He laid out a thorough organization of the proper, face-to-face tiling problem, enumerating a total of eleven cases, organized according to how many and which of the six edges have the same lengths. On page 97 he first mentions the case at hand as one of two broad cases given by certain edges being 
equal. The case of two chains of equal length edges is irreducible in the sense that if there are any further equalities then the tetrahedron becomes equilateral. The other case admits a number of specializations, or subcases, given by additional equalities of edge lengths. Then on pages 106-107 he explains how there are nine additional subcases of the first case, which he calls "grade (ii)" through "grade (x)," leaving our case as "grade (xi)."

He systematically deals with grades (i)-(x). However, he simply does not address the final case, never mentioning it again. He did not say he was omitting grade (xi) because it was similar to some of the others.

At the end he presents lists of "honeycombs in space of three dimensions" and writes, "This list may be regarded as complete, except for the possibility of some isomeric forms having been overlooked" [10, p. 15].

One would suppose that people reading the published paper would have noticed the omission, and one would suppose that Sommerville then would have been notified, and the apparent oversight corrected. That does not seem to have happened. However, if it did, then the resulting correction would seem to have been buried, without having entered into the consciousness of the mathematical community.

Perhaps no one actually read the details of the argument all the way to the end so as to notice that he had failed to account for case (xi), until Senechal's investigation in [9].

It is thus possible that he never had an argument for the final case. Certainly the argument presented here has a rather different flavor than the rest of Sommerville's analysis. The argument here is more metrical and continuous, while Sommerville's seems more combinatorial and discrete.

Now that Sommerville's classification is complete it remains to give a full account of tetrahedra that tile with no assumption that the tilings are proper, are face-to-face, or admit a face-transitive group of symmetries.

\section{References}

1. L. Baumgartner, Zerlegung des vierdimensionalen Raumes in kongruente Fünfzelle, Math.-Phys. Semesterber. 15 (1968), 76-86.

2. M. Berger, Geometry I, translated from French by M. Cole and S. Levy, Springer-Verlag, New York, 1987.

3. H. E. Debrunner, Tiling Euclidean $d$-space with congruent simplexes, in Discrete Geometry and Convexity, Ann. New York Acad. Sci., New York, 1982, pp. 230-261.

4. O. Delgado Friedrichs and D. H. Huson, Tiling space by platonic solids, I, Discrete Comput. Geom. 21 (1999), 299-315.

5. D. Eppstein, J. M. Sullivan, and A. Üngör, Tiling space and slabs with acute tetrahedra, Comput. Geom. 27 (2004), 237-255.

6. M. Goldberg, Three infinite families of tetrahedral space-fillers, J. Combin. Theory 16 (1974), 348-354.

7. M. J. M. Hill, Determination of the volumes of certain species of tetrahedrons, Proc. London Math. Soc. 27 (1896), 39-52.

8. K. P. Kiernan, Tetrahedral tilings, REU Report, Indiana University, 2003.

9. M. Senechal, Which tetrahedra fill space?, Math. Mag. 54 (1981), 227-243.

10. D. M. Y. Sommerville, Division of space by congruent triangles and tetrahedra, Proc. Roy. Soc. Edinburgh 43 (1923), 85-116.

11. D. M. Y. Sommerville, Space-filling tetrahedra in Euclidean space, Proc. Edinb. Math. Soc. 41 (1923), 49-57.

Received January 20, 2005, and in revised form February 14, 2006 and July 13, 2006.

Online publication February 5, 2007. 